\title{
Study on Numerical Simulation of Blasting Based on SPH
}

\author{
Liao Xueyan ${ }^{1,2, a}$, Jiang Yaogang ${ }^{1,2, b}$ \\ ${ }^{1}$ Sichuan Province Academy of Safety Science and Technology. Chengdu China, 610045 \\ ${ }^{2}$ Major Hazard Measurement and Control Key Laboratory of Sichuan Province. Chengdu China \\ astagger@mail.ustc.edu.cn, ${ }^{\text {b }} 113978453 @ q q . c o m$
}

Keywords: blasting; SPH; numerical simulation.

\begin{abstract}
The Lagrange, Euler and ALE are often used in simulation of explosion, but each has both advantages and disadvantages in simulation of propagation of explosion shock wave, deformation and velocity of medium. And all of them could not simulate the progress of crack propagation, so this paper proposes a new method-SPH. SPH could simulate the whole explosion
\end{abstract} from explosion, $\mathrm{r}$ crack formation and propagation to fragment.

\section{INTRODUCTION}

The blasting is a large compressive deformation, the numerical simulation based on Lagrange or Euler can not continue, because the deformation of grid is too large. The finite element method usually use erosion or remeshing to get over the difficulty. The smoothed particle hydrodynamics named SPH, which was invented by Lucy, Gingoldd and Monaghan in 1977 , was used for celestial successfully ${ }^{[2,3]}$. Then the SPH was applied to numerical simulation of underwater explosion ${ }^{[4-6]}$. Compared with the finite element and finite difference grid method, SPH method can solve the problem of large deformation and breakage. This paper uses the SPH method to simulate the process of blasting. The results show that, SPH method can the whole process simulation of blasting, the blasting explosive, explosive effect, stress and strain, cracks and expansion, crushing and throwing. Therefore, the SPH method for the simulation of blasting and blasting scheme optimization provides a new method.

\section{PRINCIPLE OF SPH}

The core of the basic idea of SPH is a kind of interpolation theory, which is based on the kernel function, the continuum is discretized into a series of mass of a particle, the kernel approximation to equation, the basic process is as follows:

1. The continuum is discretized into a series of quality SPH particle, no task connection between particles, so the SPH method without grid;

2. Field function with integral representation to approximate, in the SPH method called kernel approximation method;

3. Application support neighboring particles within the domain of the corresponding value sum to replace the integral expression of field function of field function of particle approximation; 
4. The particle approximation method is applied to all the partial differential equations of the field function related items, the partial differential equation;

5. The particles are attached to the quality, it means that with the material properties of the particles of these particles; field variables using explicit integration method to get all the particles changes with time value.

\section{SIMULATION OF BLASTING}

The SPH method was used for the typical blasting crater and throwing blasting process simulation, In order to study the feasibility by simulation of blasting process of the SPH method. The RHT material model is used on the two calculation model, the uniaxial compressive strength is $35 \mathrm{MPa}$; for ANFO explosives, the density is $930 \mathrm{~kg} / \mathrm{m} 3$, the equation of state is JWL.

\section{Simulation of blasting crater}

In order to verify the feasibility of SPH method. The simulation of typical blasting crater is be done. The physical dimension of model for rock is $6 \mathrm{~m} \times 8 \mathrm{~m}$; the geometry size of charge is $0.08 \mathrm{~m} \times 0.08 \mathrm{~m}$; the charge from the left boundary and the right boundary distance both is $4 \mathrm{~m}$, from the upper boundary $1 \mathrm{~m}$; the particle spacing is $40 \mathrm{~mm}$, as shown in Fig. 1.

The blasting crater simulated forming process based on SPH is shown in Fig. 2. As can be seen from Fig. 2: first the rock which surround the charge is crushed by the explosive stress wave after the charge explosion; then the free surface of the rock is cracked by reflective tensile stress; at the same time inside the rock cracks and gradually expand the formation fracture zone, fracture and crack mainly concentrated in the upper part, a funnel is shaped; the upper part of the rock fly out, then the explosive funnel is formed eventually.

The SPH method can simulate the whole process of the formation of blasting crater, including the explosive, The process of the rock is compressed by explosive impact, The process of rock crack, propagation, fractured, The whole simulation process is consistent with the classic theory of blasting crater. In addition the stress, speed and other important parameters of any point in the model can be calculated as shown in Fig. 3 and Fig. 4.

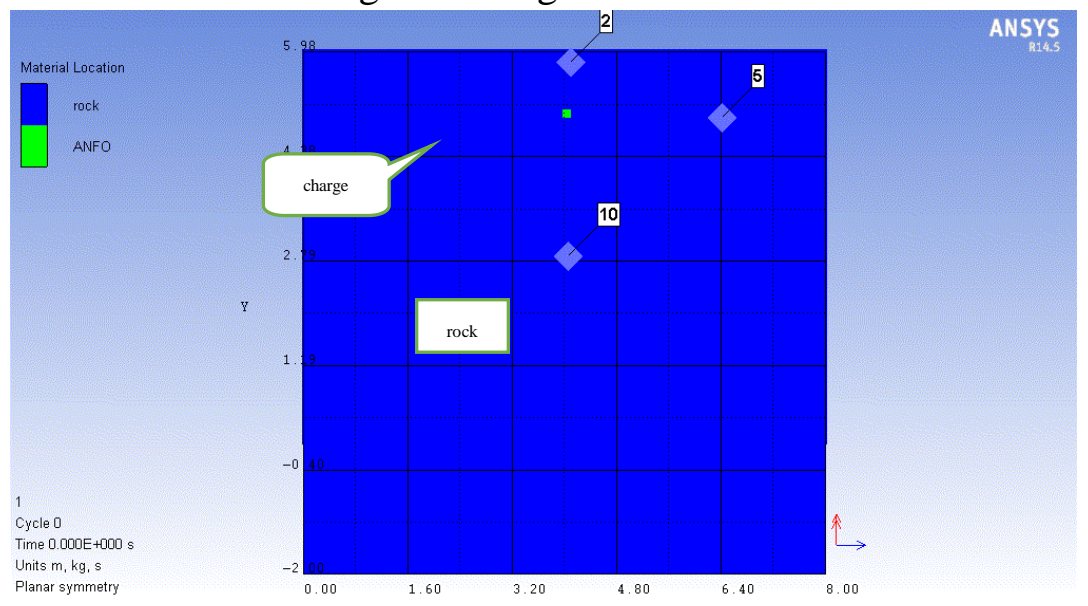

Fig.1 Model of blasting crater 


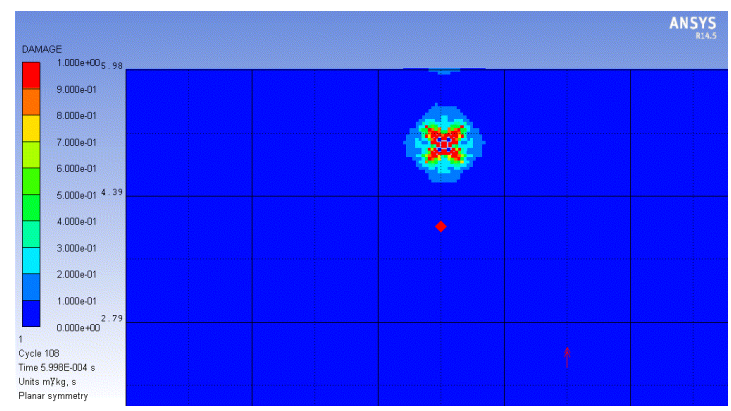

$0.6 \mathrm{~ms}$

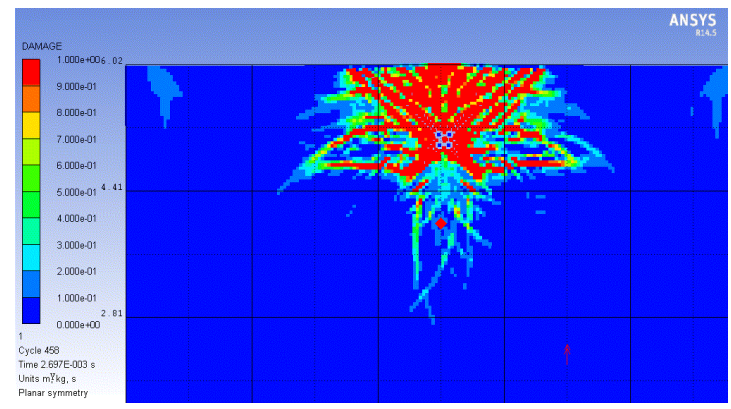

$2.7 \mathrm{~ms}$

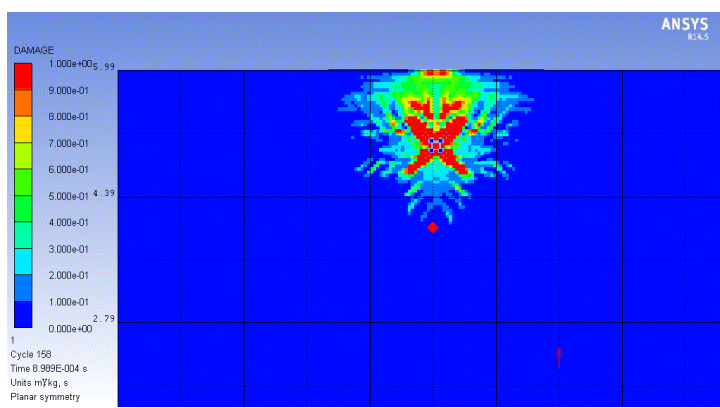

$0.9 \mathrm{~ms}$

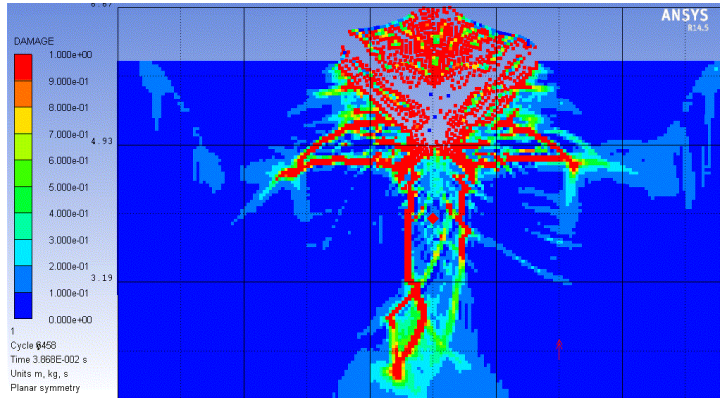

$3.9 \mathrm{~ms}$

Fig.2 Process of numerical simulation of blasting crater

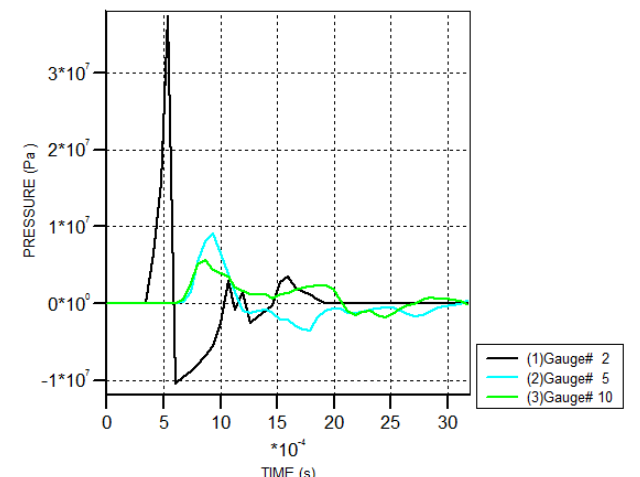

Fig.3 Stress time-history curves of particle

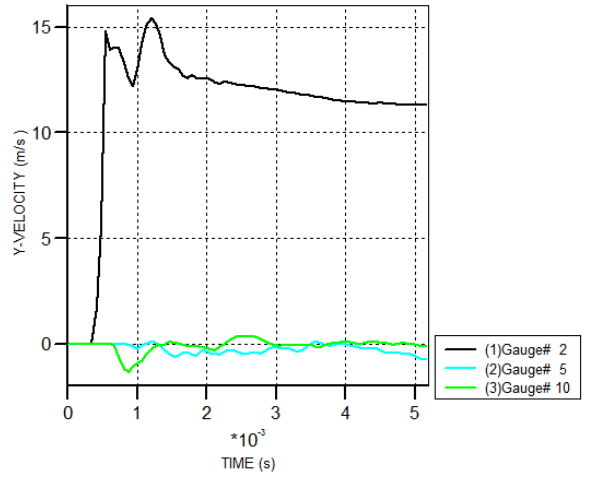

Fig.4 Velocity time-history curves of particle

\section{CONCLUSION}

(1) The explosion crater model is simulated by SPH method. The result of numerical simulation shows that the SPH method can simulate the whole process of blasting that include the breaking and the formation of explosive crater. The result is consistent with the classical theory of explosion crater.

(2) The SPH method is used to simulate the casting blast. The results indicate that SPH method can simulate the whole process of casting blasting. The simulation of blasting throwing process and engineering actual match.

(3) The whole process of blasting can be simulated by SPH methods, especially the process of crushing and throwing which traditional Lagrange method and Euler method can't be effective simulation. Therefore, SPH method for blasting fragmentation and blasting engineering research 
provides a new method.

\section{REFERENCE}

[1] Wang Xuguang. Handbook of blasting[M]. beijing: Metallurgical Industry Press, 2010.

[2] Lucy L B.Numerical approach to testing the fission hypothesis[j],Astronomical Journal.1977(82):1013-1024

[3] Gingold R A,Monaghan J J.Smoothed Particle Hydrodynamics:Theory and Application to Non-spherical stars[C].Monthly Notices of the Royal Astronomical Society.1977(181)375-389.

[4] Johnson G R,stryk R A,Beissel S R.SPH for high velocity impact computations[J].Computer Methods in Applied Mechanics and Engineering,1996,139:347-373

[5] Libersky L D,petschek A G,et al. High strain lagrangianhydrodynamics:A three-dimensional SPH code for dynamic material response[J].J Comput Phys, 1993.109:67-75

[6] Johnson G R,Beissel S R.Normalized smoothing functions for SPH impact computations[J].International Journal for Numerical Methods in Engineering, 1996,39:2725-2741.

[7] AUTODYN materials library version 6.1. 